\title{
Norois
}

Environnement, aménagement, société

$214 \mid 2010 / 1$

Le Maroc en mutation

\section{Rôle et place des migrants dans l'espace public à Agouraï}

What part and share have migrants in Agourai's public sphere?

Zoubir Chattou, Patrick Gonin et Marie-Antoinette Hily

\section{(2) OpenEdition}

1 Journals

\section{Édition électronique}

URL : https://journals.openedition.org/norois/3115

DOI : 10.4000/norois.3115

ISBN : 978-2-7535-1562-8

ISSN : $1760-8546$

\section{Éditeur}

Presses universitaires de Rennes

Édition imprimée

Date de publication : 20 mai 2010

Pagination : 41-53

ISBN : 978-2-7535-1121-7

ISSN : 0029-182X

\section{Référence électronique}

Zoubir Chattou, Patrick Gonin et Marie-Antoinette Hily, « Rôle et place des migrants dans l'espace public à Agouraï », Norois [En ligne], 214 | 2010/1, mis en ligne le 01 juin 2012, consulté le 13 janvier 2022. URL : http://journals.openedition.org/norois/3115; DOI : https://doi.org/10.4000/norois.3115

\section{(c) Tous droits réservés}




\title{
Rôle et place des migrants dans l'espace public À Agouraï
}

\author{
Zoubir Chattou \\ (École Nationale d'Agriculture), \\ Km. 10, Route Haj-Kaddourn BP S/40 - MeKnes 50001, Maroc \\ zchattou@gmail.com \\ Patrick Gonin, Marie-Antoinette Hily \\ Migrinter \\ (université de Poitiers), \\ 99 av. du Recteur-Pineau - 86000 PoITIERS \\ patrick.gonin@univ-poitiers.fr,marie-antoinette.hily@univ-poitiers.fr
}

\begin{abstract}
RÉSUMÉ
Agouraï, petite ville marocaine, située au sud de Meknès et au pied de l'Atlas, pourrait etre perçue comme un "non-lieu » de la migration internationale. Mais cette vision ne correspond pas à la réalité locale. Cet article montre le rôle et la place que prennent les migrants dans l'histoire de cette commune (10000 habitants). Les récits dont ils font l'objet à propos de la question du développement, telle que les pouvoirs publics l'envisagent, permettent de comprendre les enjeux des rapports migrants/non migrants. Objets de méfiance, de désintérêt, de jalousie mais aussi de sollicitations, les migrants sont aussi révélateurs des dynamiques sociales que connaît le Maroc aujourd'hui, notamment dans la région d'Agouraï concernée par différents projets de développements locaux.
\end{abstract}

MOTS CLÉ : migrants/non migrants - espace public - Maroc

\section{ABSTRACT}

\section{What part and share have migrants in Agouraïs public sphere?}

Agouraï is a small Moroccan town, located south of Meknes and close to the Atlas Mountains. It could be seen as a place free of international migration. But such a perception does not correspond to the local situation. This paper explores the part and share that migrants play in the history of this city of 10.000 inhabitants. Stories told about them in connection to development issues such as seen by local authorities allow understanding what is at stake between migrants and non-migrants. Seen with defiance, jealousy but also being called upon to contribute locally, migrants are also revealing the social dynamics Morocco is undergoing today, especially in the Agouraï area, through different local development projects.

KEY WORDS: migrants/non-migrants - public sphere - Morocco

Les multiples échanges entre les lieux de résidence et le pays d'origine qu'ont construit les Marocains depuis leur entrée dans la migration et les récentes formes d'organisation économiques entre les différents espaces « d'ici et de là-bas », donnent une image contrastée des engagements 
des Marocains résidant à l'étranger dans les dynamiques de développement des régions d'origine (Charef, 2005; Charef et Gonin, 2005 ; Arab, 2009). Si historiquement les zones de départ ont été le sud (le Souss) et le nord (le Rif), deux régions pauvres caractérisées comme périphériques ${ }^{1}$, d'autres espaces d'émigration vont ensuite être concernées (les régions d'Oujda et de Nador notamment) (Berriane et Hopfinger, 1992; Chattou, 1995). La migration ne s'est cependant pas étendue à l'ensemble du Maroc et certaines zones géographiques n'ont connu qu'un faible flux de départ dont la province d'El Hajeb au sud de Meknès (fig. 1).

Malgré l'abondante littérature traitant de l'émigration ${ }^{2}$, l'on constate que les réalités migratoires à grande échelle topographique sont peu traitées, tout comme les zones où la densité migratoire est faible. En outre les rapports entre les «villageois » et leurs « émigrés-immigrés » (Charef et Gonin, 2005), les migrants et circulants n'ont fait l'objet que de très peu d'enquêtes géographique et sociologique (Schaeffer, 2004). On signalera cependant les recherches menées par T. Lacroix (2005a) qui montrent l'implication des migrants dans le développement des villages d'origine dans la région du Souss-Massa-Draa. L'auteur relève les tensions entre migrants et villageois d'une part, qu'il qualifie de culturelles mais aussi de politiques, et d'autre part la frilosité des pouvoirs publics locaux marocains à prendre en compte les différents rôles des migrants dans des projets de développement (p. 204). Notre projet dans cet article est de poursuivre cette réflexion sur la base d'une enquête de terrain menée à Agouraï (province d'El Hajeb) où il s'est agi de prendre la mesure des liens sociaux qui se maintiennent ou s'effritent entre ceux de là-bas et ceux d'ici ${ }^{3}$.

En explorant les représentations des Marocains à l'étranger à partir des discours que tiennent les notables, les militants associatifs impliqués dans des programmes de développement intéressant le «village ${ }^{4}$ » et la province, ainsi que dans les récits des habitants et de jeunes enfants, nous rendons compte de la façon dont sont catégorisés les migrants et comment s'expriment, malentendus, revendications et aspirations au changement économique et social quand il est question de leur place et de leur rôle dans un espace villageois ${ }^{5}$ " en développement ». Pour examiner ces questions, nous exposerons d'abord le contexte agouranais pris comme terrain et objet de discours pour aborder ensuite les débats qui ont lieu dans l'espace public villageois ${ }^{6}$ à propos de la rénovation de la Casbah, et plus généralement des projets de développement économiques et sociaux locaux et de l'implication des migrants dans ces initiatives au prisme des représentations que les villageois s'en font.

1. La migration a d'abord été de proximité (l'Afrique de l'Ouest pour le Souss et l'Algérie pour le Rif), avant de se diriger vers les entreprises Françaises et Belges qui ont largement fait appel à cette main-d'œuvre rurale et peu qualifiée (Noin, 1970).

2. Dans la base MigrinterNet (fonds documentaire du laboratoire Migrinter), nous avons identifié 721 notices correspondant à des références bibliographiques de documents en langue française sur le thème des migrations en relation soit avec le pays (Maroc), soit avec la population (Marocains). Cependant, le nombre de références diminue fortement (252) quand il s'agit exclusivement de documents qui traitent des migrations au Maroc (thématiques : politiques migratoires, droits, insertion / intégration...). Le nombre de références concernant les Marocains est de 469 : il signale des documents qui traitent de la population marocaine émigrée par exemple en France (177), en Belgique (52), en Espagne (44) ou en Italie (17). On peut estimer à la lecture de ces résultats que la base MigrinterNet donne approximativement un tiers de la production éditoriale sur le sujet au pays, et les deux tiers sur la population. Dans le cadre d'une recherche en cours - programme Volubilis -, seule la moitié du volume global de la production est identifiée. En effet, la base de données que nous mettons en place, après les derniers contrôles et la réindexation, contiendra certainement un peu plus de 1500 références francophones. L'explosion récente du nombre de publications sur ce pays s'explique en partie par la multiplication des travaux d'auteurs marocains.

3. Comité volubilis Action $n^{\circ} \mathrm{AI} n^{\circ}$ : MA/06/158 : «Initiatives socio-économiques des Marocains résidents, à l'étranger, dans les sociétés méditerranéennes »

4. Nous avons respecté l'emploi du mot «village » par les acteurs locaux : il désigne à la fois la ville d'Agouraï et les douars de cette commune.

5. Parmi les nombreux thèmes abordés lors des entretiens, nous avons privilégié celui de la gestion locale, des initiatives de développement et du rôle des migrants dans ces initiatives.

6. Nous n'utilisons pas le terme «d'espace public » dans le sens habermasien mais dans le sens de la possibilité et de la capacité d'une expression critique publique. Nous rejoignons par là les propositions de J.-N. Ferrié (1999, p. 76) quand il écrit « Disons tout de suite aussi que j'entendrai ici "espace public" au sens de "parler en public" et, plus exactement, "parler en public de politique"». 


\section{Le « village » d'Agouraï et les enjeux locaux du développement}

La municipalité d'Agouraï se situe au centre du Maroc (fig. 1), entre deux grandes métropoles urbaines : Fès $(75 \mathrm{~km})$ et Meknès $(25 \mathrm{~km})$. Elle appartient à la province d'El Hajeb ${ }^{7}$. Elle est également le centre urbain du cercle administratif d'Agouraï (zone de montagnes et de plaines) qui inclut cinq communes rurales (Tamchachat, Ait Ouikhalfen, Jahjouh, Ras Jerry et Aït Yaazem). Cette province s'étale sur une superficie de $2209 \mathrm{~km}^{2}$ et comprenait au dernier recensement (RGPH 2004) une population de plus de 216000, soit 10,1 \% de la population de la Région de Meknès/Tafilalet.

Fondée par le Sultan Moulay Ismail à la fin du XVIII ${ }^{\mathrm{e}}$ siècle, la cité d'Agouraï assurait des fonctions multiples :

- protection militaire contre les attaques des tribus rebelles des alentours;

- contrôle des richesses agricoles et du commerce caravanier entre le sud du Maroc et la capitale Ismaélite de Meknès;

- lieu d'emprisonnement des pirates (plusieurs descendants de portugais, semblent-ils, vivent encore à Agouraï avec un nom arabisé).

Cette fortification a favorisé la naissance d'une petite ville aux confins des tribus du Moyen Atlas peuplée d'Arabes, de Berbères, d'Africains venant du sud du Sahara, enrôlée par le sultan dans son armée, de réfugiés et de prisonniers dont une partie a fini par s'établir à Agouraï. Cette casbah comme nous le verrons est devenue centrale dans les stratégies locales. À la fois lieu d'histoire et d'identité, elle est aussi un enjeu politique et fait l'objet de controverses dans l'espace villageois à propos de ce qui est nommé « le développement territorial ».

La carte de la population de la province d'El Hajeb nous renseigne sur l'émergence du centre urbain d'Agouraï (fig. 1). Il occupe la quatrième position en termes de densité de population après la ville d'El Hajeb (siège de la province), Aïn Taoujtat et Sabaa Ayoun. Elle est à l'image de celle du Maroc : les moins de 15 ans représentent près de $30 \%$ de la population et les $2 / 3$ de celle-ci ont moins de 35 ans. Par contre, moins de $10 \%$ ont un âge qui dépasse les 59 ans. L'indice synthétique de fécondité à Agouraï est de 2 enfants par femme. Il suit une baisse progressive par rapport au recensement général de 1994. Aussi nous observons un recul très significatif de l'âge du mariage et une généralisation des mariages tardifs. Ceci exprime des changements démographiques qui affectent le Maroc d'aujourd'hui, y compris dans ses zones rurales et semi-urbaines. L'âge moyen au premier mariage est de 28,5 ans et il se répartit inégalement selon les sexes. Les hommes se marient à un âge d'environ 31 ans contrairement aux femmes qui se marient entre 26 et 27 ans. Si nous remarquons un relatif équilibre entre les deux sexes en ce qui concerne la population des mariés, le déséquilibre est plus prononcé au niveau des célibataires. Les femmes demeurent moins célibataires que les hommes. Aujourd'hui avec ses 2724 ménages, soit 13291 habitants (49,2\% d'hommes et $50,8 \%$ de femmes), la municipalité connaît une croissance démographique par son solde naturel positif et par l'apport de population rurale (données RGPH, 2004). Par ailleurs, l'écart enregistré entre les hommes et les femmes pourrait être la conséquence des migrations masculines à l'intérieur comme à l'extérieur du Maroc.

Le taux d'activité de la population d'Agouraï est de $35 \%$. 15,3\% des femmes ont une activité économique génératrice de revenu, contre $55 \%$ pour les hommes. Les femmes sont davantage dans le travail domestique et familial. Une même inégalité se manifeste au niveau du taux d'analphabétisme selon les sexes : les hommes d'Agouraï sont à $34 \%$ analphabètes contre 57,2 \% de femmes. Bien que la moyenne des deux sexes soit relativement supérieure $(46 \%)$ à celle au niveau

7. La province est une entité territoriale qui se compose de pachalik pour les villes et de cercles administratifs pour les espaces ruraux. Le gouverneur est la plus haute représentation de l'État au sein de la province. Le caïd coiffe en général une commune rurale et le Pacha une municipalité. Les communes sont la représentation politique de la population. Il y a aussi un conseil provincial qui est une organisation représentative de l'ensemble des communes dans la gestion du territoire provincial. Si l'organisation administrative provinciale s'inspire du modèle français, il existe tout de même une différence de taille. Les communes au Maroc tant rurales qu'urbaines sont sous la tutelle de l'État. Le Gouverneur se retrouve de droit l'ordonnateur financier ce qui complique la démarche d'autonomie des politiques communales. 


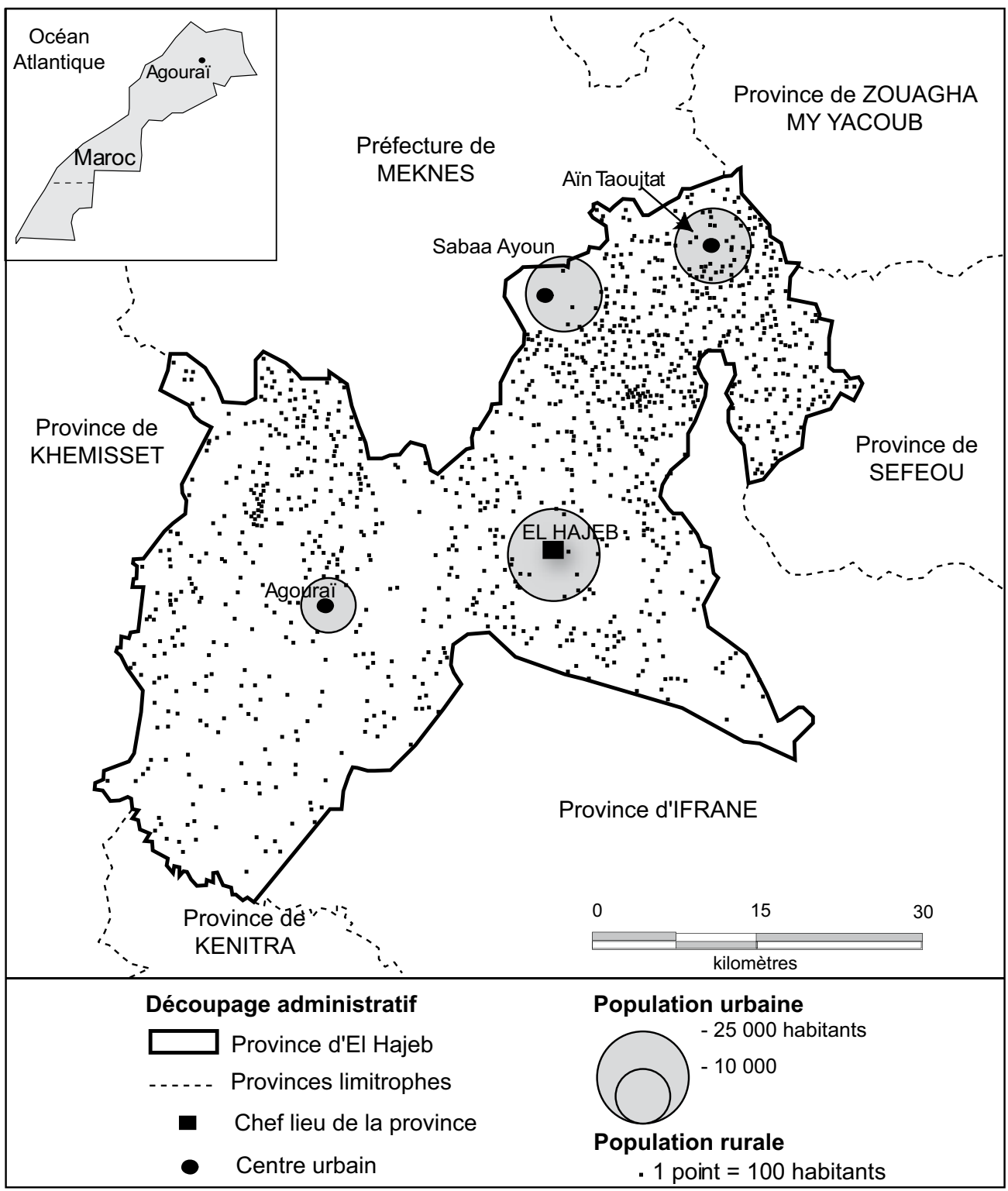

Figure 1 : Localisation de la ville d'Agouraï et répartition de la population de la Province d'El Hajeb (source : RGPH, 2004)

Localisation of Agouraï town and distribution of the population of El Hajeb Province

national $(43 \%)^{8}$, elle demeure néanmoins très discriminante notamment au niveau de l'accès des femmes et des hommes à l'emploi. Les femmes et les hommes qui se déclarent actifs sont indépendants à 31,5\%, les femmes étant essentiellement occupées par des activités dans la sphère domestique (petits élevages, tapis, artisanat...). Les hommes qui déclarent avoir un commerce (épicerie, boutique et garage, kiosque...) ou une activité artisanale représentent environ le 1/5 de la population active (RGPH 2004). Plus de la moitié de la population active d'Agouraï occupe un 
poste de salarié dans le secteur privé et particulièrement dans l'agriculture. On remarque aussi que le développement d'une agriculture "moderne » a entrainé une féminisation des emplois dans ce secteur, les femmes étant plus recherchées par les employeurs pour la cueillette des fruits notamment.

La population en migration est difficilement chiffrable, tout comme les revenus qu'elle génère. Mais d'après nos enquêtes, il semblerait que 400 familles originaires de la commune sont à l'étranger. Si les premières générations qui ont émigré en France, en suivant les itinéraires des ex-colons anciennement établis dans la zone, ont d'abord mis leurs économies dans l'immobilier à El Hajeb ou à Meknès, les migrants récents en Espagne, en Italie et en France ont eux investi dans la ville d'Agouraï. La plupart des investissements se sont faits dans le bâtiment, et dans une moindre mesure dans l'agriculture, le petit commerce et les services. Ces constats ne sont pas surprenants car à Agouraï comme dans les régions de forte émigration, les immigrés investissent d'abord dans le logement en vue du retour même si souvent celui-ci ne se réalise pas. Et la dimension symbolique de ces investissements est bien connue : elle est la marque visible d'une réussite sociale ${ }^{9}$ alors que les maisons construites ne s'animent qu'un mois par an. D'ailleurs la ville d'Agouraï et sa population subissent cette urbanisation croissante due aux migrants sans qu'elle ne soit considérée comme une amélioration du «standing urbain ». De plus elle a provoqué une spéculation foncière qui contribue à l'exclusion de larges couches de la population locale dans l'accès aux logements.

\section{Casbah eT Marché COUVERT : ObJets de déSACCORD}

Les élus locaux affichent une volonté de structurer la ville tout en développant son attractivité. La restauration de la casbah et la création d'un quartier de métiers vont dans ce sens. Mais alors que cette restauration est présentée par les élus comme une priorité, certains militants associatifs sans s'y opposer radicalement, restent cependant sceptiques devant un tel projet qu'ils jugent soit inadapté aux besoins d'Agouraï soit comme une réalisation dont les conséquences ont été mal anticipées. Plus encore, la rénovation de la casbah cristallise des visions différentes du développement dans cette région du Maroc pauvre où les infrastructures (assainissement, électrification, voirie, construction de la route de Marrakech/Kénitra) restent encore largement inachevées et à l'état de projet. Et c'est bien sur ces questions que les désaccords sont les plus visibles.

Cette initiative inscrite dans l'agenda $21^{10}$ date d'une dizaine d'années. Elle a impliqué de nombreuses concertations entre le conseil provincial, la municipalité et les services de l'aménagement du territoire. Le coût financier représente près de 600 millions de Dirhams (Dh) (environ 60 millions d'euros) financés pour partie par le Conseil Régional, le Conseil Provincial, le Ministère de la Culture et les Nations Unies dans le cadre d'un programme de valorisation du patrimoine. Aujourd'hui la réfection des murs d'enceinte est terminée, mais de nombreux travaux restent à entreprendre pour aménager l'intérieur et surtout rendre attractif ce patrimoine dont beaucoup espère qu'il participera au développement d'un éco-tourisme, thème largement développé par certains élus, dont nombre d'entre eux considèrent qu'il pourrait devenir le levier du décollage économique du «village ». Mais la casbah est encore habitée par des familles à très faibles revenus (environ 1200 personnes) et l'« évacuation » d'au moins une partie est d'ores et déjà prévue afin de valoriser le patrimoine, ce qui ne va pas sans soulever des polémiques. Pour quelques militants associatifs, cette démarche est anti-touristique ( ses habitants font la casbah ») alors que pour des élus, il s'agit d'aménager des hébergements touristiques et de favoriser l'installation de commerces artisanaux.

9. Une compétition s'opère entre les familles non seulement sur le fait de construire mais aussi sur des signes de prestige comme la taille de la maison ou les matériaux utilisés (faïences, accessoires, peintures...).

10. Il s'agit d'un programme d'action définissant les objectifs et les moyens de mise en œuvre du développement durable à l'échelle des collectivités locales. Il est élaboré en concertation avec l'ensemble des acteurs locaux. L'Agenda 21 local représente dans ce sens un outil opérationnel du développement durable, tout comme il intègre, par son approche, les principes de la gouvernance locale [http://www.agenda2 Imaroc.ma/]. 
Zoubir Chattou, Patrick Gonin, Marie-Antoinette Hily

Un autre terrain de discussion concerne le marché couvert au centre d'Agouraï. Pour l'instant fermé, son ouverture est prévue, mais sans que l'on donne de date précise. Il devrait prendre la place du marché ouvert situé à la sortie du village et dont la pratique n'est guère aisée les jours de pluies. Selon le président de l'association Al Houda, «la construction du marché a été faite en pure perte, il ne correspond pas aux besoins de la population ». Mais cet avis est aussi lié à son financement dont beaucoup soupçonnent qu'il a été mis au service d'entreprises soutenues par quelques élus de la municipalité. Mais pour M, élu au conseil provincial, si « Agouraï se développe c'est grâce au conseil municipal actuel, les élus sont jeunes, ils ont des idées et puis il y a des programmes ». Et c'est bien autour de la définition des objectifs de ces "programmes » que les dissensions apparaissent, notamment entre élus locaux et «nouvelles élites », mais aussi au sein même des structures représentatives.

De manière générale, l'ensemble des projets à l'agenda des instances locales ou provinciales ne va pas sans déclencher des discours soupçonneux quant à l'attribution des marchés et aux conditions d'exécution de ceux-ci.

\section{DeS APPROCHES DIFFÉRENTES DU « DÉVELOPPEMENT » : TOURISME VERSUS AGRICULTURE}

Les divergences qui se font jour quant aux choix à faire en matière de « développement » (ce terme est largement utilisé par les « acteurs locaux ») témoignent de conceptions bien différentes de celui-ci dans une région comme Agouraï où l'agriculture reste dominante. Ainsi, certains de nos interlocuteurs, militants associatifs, font porter leur critique sur les priorités locales en matière de développement. Ils estiment qu'elles devraient se situer dans la croissance de la production agricole justifiée par le fait simple que les emplois sont dans ce secteur (les terres d'Agouraï sont cultivables et irriguées), que les chefs de famille n'ont pour la plupart que cette ressource pour vivre et qu'enfin, ils sont nombreux à travailler comme journalier sur des grands domaines pour 60 dirhams (soit environ 6 euros) par jour alors qu'ils pourraient avoir un revenu supérieur sur leurs propres terres s'ils pouvaient les exploiter. L'agriculture d'un côté et la valorisation du patrimoine pour le bénéfice d'un éco-tourisme de l'autre, telles pourraient être l'enjeu du débat. Mais sousjacent à ces désaccords sur l'avenir d'Agouraï, on trouve des visions de l'intérêt général qui vont jusqu'à s'opposer (l'amélioration des infrastructures serait un de leur point commun cependant). Mais bien plus que des divergences politiques entre « élus » et militants associatifs, ce sont plutôt des divergences idéologiques entre de jeunes acteurs qui ont fait des études et qui se sentent impliqués dans la gestion locale, qu'ils aient une responsabilité municipale ou pas. Certains ont une vision du développement qui passe par le tourisme de montagne (il s'agit de "vendre la nature » selon leur expression) et le patrimoine (musique, produits du terroir, traditions). Pour d'autres le « développement » implique de prendre en compte les ressources locales et les besoins d'une population à faible revenu. Les premiers ont souvent des compétences techniques et s'inscrivent dans « une culture du projet », les autres ont, au moins pour les leaders, connu l'émigration mais leur inscription dans le « village » est soutenue par un discours qu'ils disent plus proche des réalités locales. Ces « gens de terrain », bien insérés localement, font tous partie d'une génération qui tente de s'inscrire dans un Maroc qui n'est plus considéré comme « en voie de développement » mais un Maroc qui a à jouer un rôle au sein des pays méditerranéens.

Nous sommes donc devant un débat public de type nouveau au sein du monde rural marocain. Le développement des associations a contribué à la construction de nouvelles élites locales souvent bien formées et qui rivalisent avec les élites traditionnelles (Dumont, 2007). Chacune s’inscrit dans des registres de légitimité et des logiques d'actions particulières. La contestation, l'opposition et la force de proposition ne sont plus le seul monopole de l'élu, ni des autorités représentant l'État, la « société civile». Et surtout, ses représentants commencent, certes encore timidement, à entrer dans le jeu de la concertation locale. Tout se passe comme si l'action associative devenait un laboratoire de formation de nouvelles élites en compétition dans le champ de la légitimité politique à l'échelle locale et du contrôle du pouvoir communal. Dans cette logique, la constitution récente 
d'associations à Agouraï peut être considérée comme une nouvelle forme de mobilisation mettant à l'épreuve les initiatives des pouvoirs en place. Mais elles peuvent être aussi considérées comme un analyseur des changements sociaux que connaît le « village », et plus généralement la région d'Agouraï, et comme une réponse aux problèmes du « développement ».

\section{COMPÉTENCES ASSOCIATIVES}

L'association « Al Houda pour le développement, la culture, la solidarité » a été créée en 2004. «Sa constitution est le fruit du hasard » nous dit son actuel dirigeant, un enseignant de français qui s'est entouré de proches pour la constituer. Le vice-président réside et dirige une clinique à Carpentras. Originaire d'Agouraï, il est très actif dans les initiatives que prend l'association, de même que le secrétaire général immigré en Virginie où il poursuit des études grâce à une Green Card gagnée à la loterie. Nombre de membres de sa famille ont occupé des responsabilités au bureau, dont sa femme et sa fille. Sur les murs du local de l'association une affichette signale au visiteur les partenariats que l'association a noués. Parmi les partenaires nationaux, on relève : l'Entraide Nationale, le Secrétariat d'État à la Famille à l'Enfance et aux Personnes Handicapées, l'Initiative Nationale de Développement Humain, la Santé Publique, la province de El Hadjeb, les communes du cercle d'Agouraï, l'association Horizons Ouvertes-Temara, le réseau Agora-Maroc et l'IPDF-Fès. Parmi les partenaires internationaux, on relève : l'Ambassade du Japon - Rabat, la Coopération Japonaise à Rabat, l'Oxfam Québec-Montréal-Canada, l'association franco-marocaine d'Avignon et l'association Mohagir de Carpentras - France. Cette énumération montre la diversité des collaborations qu’a su nouer le président pour répondre aux objectifs de l'association essentiellement consacrés, soulignet-il, aux besoins de la population locale : insertion de la femme rurale, coopérative féminine pour l'élevage des caprins, centre Internet et initiation à l'informatique pour les jeunes, construction de la maison de la jeune fille, structure para-médicale pour les jeunes accouchées. Et les projets ne manquent pas, qu'ils concernent la plantation de 50000 caroubiers dans la région ou la création d'une classe pour les handicapés etc. Si le président nous dit travailler sans l'aide des élus locaux, il multiplie par contre les contacts et les projets avec l'État marocain tout en s'appuyant sur des immigrés qui participent de la même idéologie en matière de développement. Elle se résume à l'idée que « le développement passera par les habitants et leurs besoins » et c'est «l'agriculture qui fera bouger la ville », ainsi que le développement d'activités innovantes génératrices de revenus.

Dans l'association Al Houda les demandes de financement des projets se font en dehors des autorités locales et son président précise : «Les initiatives privées président aux financements des associations [...], chez nous chacun a ses propres initiatives [...], les élus ont peur que je choisisse de me présenter aux élections et que je prenne leur place, c'est un problème politique [...], ce que j'ai gagné (avec l'association) c'est des contacts [...], je connais beaucoup de ministres [...] c'est tout. Dans le projet avec les Japonais, j'ai la possibilité d'embaucher sept personnes [...] comme l'approche genrée est à la mode, il y aura cinq filles et deux garçons. "Si le président s'est engagé dans la gestion de l'association c'est aussi parce que les pratiques des élus ne lui ont jamais paru convaincantes : «Il faut que ce soit les gens qui décident pour eux [...]. Avant de faire quoique ce soit, on se concerte [...], sans la route il n'y aura pas de commerces [...]. On fait venir un spécialiste de l'électricité pour vous parler des routes, c'est le surréalisme [...]. Il n'y a pas de politique cohérente, c'est incompréhensible, mais c'est comme ça [...]; la plupart du temps on fait appel à des experts avec qui l'on peut gagner de l'argent [...] c'est ce qui se fait sous la table qui entrave... »

Dès lors, quand on parle de « développement » et de modernisation du milieu rural, nos interlocuteurs nous renvoient aux situations politiques locales et aux élus dont la réputation se construit sur leurs compétences, la confiance qu'ils inspirent et en définitive sur leur crédibilité, bien plus que sur leur légitimité donc. En fait, ce qui est décrit c'est la difficulté à ordonner et rationaliser des investissements au niveau local sans entrer dans des jeux d'alliances. Mais le paradoxe est que dans une société dépeinte comme tiraillée entre allégeance aux pouvoirs «traditionnels »et inscription dans une modernité qui exige « des initiatives cohérentes », les associations peuvent 
Zoubir Chattou, Patrick Gonin, Marie-Antoinette Hily

jouer leur propre carte et mobiliser des partenaires pour, sinon colmater les brèches des ratés des pouvoirs locaux, du moins prendre des initiatives pour soutenir et aider les plus défavorisés.

La place des immigrés dans ces jeux politiques est difficile à comprendre, mais ce qui apparaît est, là encore, une série de malentendus. À la fois recherchée, de façon singulière ou ignorée mais de façon hésitante, tout se passe comme si nos interlocuteurs ne savaient la situer. D’où les indignations et les jugements moraux divergents à leur égard dans une société où ils comptent mais où on se propose de faire sans eux.

\section{Le « village » et « ses » immigrés}

À Agouraï, la génération des immigrés des années 1970 a participé à la transformation du bourg, ou du «village » comme il est nommé par ses habitants, en construisant des maisons bien visibles dans un quartier-lotissement dénommé « le quartier des immigrés ». Pour l'un de nos interlocuteurs, les immigrés « consomment du ciment et des briques et vont faire leurs courses à Marjane » (supermarché à l'entrée de Meknès). Non sans humour, il nous signifie par là que leur participation à la vie économique locale est réduite (les taxes payées par les immigrés ne représenteraient qu'entre 0,1 et $0,3 \%$ du budget total de la population). Il estime également que leur projet est de préparer leur retraite alors que la maison est construite et que certains tirent un revenu de quelques petites fermes et de petits commerces et cafés tenus par des membres de leur famille, et dans le meilleur des cas de la possession d'une maison sur la rue principale ${ }^{11}$. "Le vrai impact de l'immigration sur la région, vous allez voir, c'est des belles maisons dans un quartier réservé aux immigrés [...]. Ils ne donnent pas d'argent pour construire une bibliothèque, des écoles. " Et même quand ils sont dans des logiques de soutien aux solidarités locales, ils se retrouvent autour de projets de construction de mosquées et d'actions caritatives.

Mais, à côté de ce panorama général, deux ou trois immigrés sont présentés comme ayant apporté au village, tel ce propriétaire d'une station-service qui est fils d'immigré en Belgique et qui par le jeu des clivages locaux s'est retrouvé président de la commune d'Agouraï. Ce jeune président appartient à cette nouvelle élite construite au cœur de l'action associative et qui, porté par des enjeux locaux, s'est vu investi d'une responsabilité politique. Il a ainsi permis à de jeunes techniciens, cadres communaux, jeunes licenciés de l'université au chômage, classes moyennes (instituteurs, professeurs), etc. d'avoir une expérience d'élus au service de l'intérêt général. Aujourd'hui fortement contesté, il tente de se situer entre des héritages politiques «traditionnels » (lui-même fait partie des notabilités traditionnelles locales) et des courants « modernes » en convergence avec les aspirations de la jeunesse.

\section{DeS MigrantS MOdèles : « UNE AFFAIRE DE MENTALITÉ »}

M., membre du conseil provincial et résident à El Hajeb, distingue deux types d'immigrés : "Celui qui a dans la tête son pays natal, et là c'est une valeur ajoutée. Ils apportent de l'argent. Ce sont des migrants qui sont des entrepreneurs, ce sont des "ambassadeurs", ils sont en relation avec le conseil municipal. » Il nous cite l'exemple de ce boucher qui « fait bouger la ville » par ses investissements ou cet autre qui a constitué une société d'intérim en France pour construire un complexe hôtelier, dont l'exploitation aura des retombées financières importantes pour la commune. Il y a encore celui qui a «une station essence, des logements qu'il loue, dont les bâtiments du Crédit Agricole [...]. C'est quelqu'un qui cherche quelque chose, il a une expérience de l'extérieur, il fait son retour, c'est quelqu'un qui pense à son pays, à sa ville, il a une personnalité, il pense aux autres, il a une moralité». Ce sont donc « des personnalités » peu nombreuses qui auraient « gardé un attachement au pays » et qui s'investiraient dans sa gestion.

11. Les locaux de la mairie de Tamchachat, une des 5 communes rurales du cercle administratif d'Agouraï, sont loués à un immigré. 
L'autre type de migrant évoqué est celui qui «même riche gaspille son argent, fait des fêtes quand il vient en vacances mais qui ne fait rien pour la commune ». À ce type de migrants s'ajoute celui que l'on nous décrit comme ne participant pas à la vie de la commune. S'il investit c'est pour sa famille, dans la construction d'une maison, dans un lotissement souvent et dans le meilleur des cas dans l'achat d'appartement de rapport ou encore dans un commerce que tient un membre de sa famille. Pour M, les maisons sont occupées occasionnellement et ne sont en rien une plus value pour les communes. À l'inverse, des élus de la province pensent que c'est à eux d'inciter les immigrés à investir dans les communes. En 2007 une rencontre a été organisée dans ce sens dans une commune voisine d'Agouraï. Mais si du point de vue des autorités la question se pose de favoriser (ou attirer) les investissements, ils conviennent aussi que les problèmes bureaucratiques et administratifs sont souvent un obstacle à la concrétisation de projets éventuels.

Le président de l'association Al Houda lui opère des distinctions entre ceux «qui sont en France et en Belgique [et qui] ne sont plus des immigrés, ils sont Belges ou Français, ils ont la mentalité belge ou française » et les «fidèles » au village, comme le vice-président qui offre des ordinateurs pour les jeunes, distribue des cartables ou équipe en lit la maison de la maternité. Mais de façon générale les émigrés ne sont guère considérés comme une ressource pour le budget des communes. Non seulement ils sont peu présents, mais de surcroît et pour la majorité, leurs moyens ne leur permettent pas de participer aux « investissements solides» dont a besoin la population. Un de nos interlocuteurs, militant associatif, a une autre interprétation de la place et du rôle des immigrés à Agouraï. Il considère que la différence n'est pas « entre migrants riches et migrants pauvres » mais qu'il s'agit plutôt d'une "affaire de mentalité ». Et sous ce terme il entend non seulement leurs manières d'être pendant les vacances, leurs façons de se faire remarquer etc. mais aussi l'intermittence de leur présence qui ne les inscrit évidemment pas dans le cours des choses qui se déroulent au village. De plus et sans pourtant se tenir à l'écart, certains immigrés considèrent "qu'il n'est plus possible aujourd'hui d'investir avec les bénéfices de l'immigration (Monsieur D. propriétaire d'un restaurant-snack en bord de route). Et si certains comme Monsieur D. ont d'après lui réussi à rentabiliser un parcours migratoire «là-bas » en France et «ici » au Maroc en montant des commerces notamment, il mesure les changements intervenus depuis 2002 : «C'est un nouveau Maroc, il y a des chantiers du nord au sud. » Et «ces chantiers » comme il dit non sans fierté, se font sans les immigrés.

\section{« INTÉGRER » LES MIGRANTS}

À Agouraï cependant les initiatives en direction des immigrés ne manquent pas, mais leurs finalités du moins pour les organisateurs sont d'abord culturelles. La création de la journée du migrant (Youm al mouhajir ${ }^{12}$ ) le 10 août par les institutions publiques offre l'occasion aux différents départements de l'État et aux associations de le célébrer à leur manière. L'objectif explicite est de renforcer les liens entre le Maroc et les Marocains résidant à l'étranger mais aussi de les inciter à devenir des acteurs économiques et sociaux au sein de leur territoire d'origine. D'ailleurs les banques et les promoteurs immobiliers s'activent pour prendre place sur ces espaces - vitrines pour vendre leurs produits.

Ainsi, à Agouraï, l'association « Initiative France-Maroc », très proche de Al Houda, a organisé un festival qui a duré deux jours et qui a rassemblé « ceux du village ». La majorité des immigrés en vacances ont participé aux différentes animations (jeux de boule, spectacles). Ces moments de retrouvailles, qu'on nous signale comme importants pour tous, sont nouveaux à Agouraï. Dans cet espace et ce moment festif, il s'agit pour les organisateurs de maintenir des liens (ou de recréer) entre ceux d'ici et ceux de là-bas mais surtout de faire que les ruraux agouranais aient accès à « leur culture » et à leur patrimoine culturel. Il s'agit aussi en « animant le village » d'occuper les jeunes,

12. Les termes vernaculaires utilisés dans cet article sont définis dans un lexique à la fin de ce numéro spécial Maroc de la revue Norois. 
Zoubir Chattou, Patrick Gonin, Marie-Antoinette Hily

et plus généralement, comme on nous le présente, de contribuer «au développement humain » et de « rendre active la population locale».

\section{UNE ENQUÊTE À L'ÉCOLE : UN « VILLAGE » PEU ATTRACTIF}

Les jeunes ${ }^{13}$ que nous avons rencontrés n'ignorent ni la fermeture des frontières des espaces européens ni le besoin des populations locales qui considèrent la migration comme un droit à la survie, voire une continuité entre l'ordre colonial et post-colonial. Ainsi les jeunes avec qui nous avons échangé, à l'exception d'un seul qui dit vouloir rester dans son pays, gardent l'espoir de partir à l'étranger. Les discours convergent pour souligner le manque d'avenir professionnel dans un pays où le taux de chômage est important. Le «village » est aussi faiblement attractif pour une population jeune qui a accès à Internet, à la parabole et qui aspire à un style de vie urbain meilleur.

Confrontés aux départs des proches vers l'étranger depuis leur jeune âge, les enfants se retrouvent malgré eux complètement intégrés dans ce processus migratoire considéré comme un phénomène d'adultes. Ils le vivent souvent en silence à travers les discours et les pratiques des parents, des frères et soeurs, du voisinage et des relations de parenté en général. Ils sont très fortement affectés par les implications qu'a la migration sur leur vie. Fragilisés par leur situation de mineurs qui les rend dépendants des adultes, les enfants ne sont cependant pas dans l'ignorance de ce que représente la migration.

Les élèves valorisent les bienfaits de l'école ${ }^{14}$ et disent explicitement que c'est un moyen d'accéder au savoir. Mais quand ils comparent les jeunes diplômés qui ne trouvent pas d'emploi au Maroc et ceux en migration qui n'ont pas fait d'études (considérés par les enfants comme analphabètes) alors leur jugement sur l'école se nuance. Ainsi nous disent les enfants : «Mon cousin n'a jamais été à l'école mais il a une belle voiture et il est en train de construire une maison grâce à son émigration en France "; "L'école t'apprend à lire et à écrire mais elle ne te permet pas de réussir dans ta vie. L'étranger t'offre une occasion de réussir ta vie "; "Si j'ai à choisir entre rester à l'école ou partir à l'étranger, je choisirais l'étranger, pourquoi perdre mon temps si j'arrive à faire ma vie sans l'école »; "C'est bien d'avoir un diplôme si on part à l'étranger après, on peut trouver un meilleur travail contrairement à ici. À Agouraï, certains ont des diplômes mais ils sont encore au chômage. »

Ce qui s'exprime dans ces jugements c'est d'abord l'idée souvent répandue que le bien-être matériel n'est accessible que dans la migration qui « en plus » permet d'accéder à une réussite sociale $^{15}$. L'attitude ostentatoire des migrants, durant les vacances notamment, n'est pas sans conséquences sur les rêves d'avenir des élèves et la place de l'argent au centre des discussions des adultes nourrit leur imagination.

Pendant les vacances c'est aussi les cousins, cousines de leur âge, les «vacanciers débarquant au bled $^{16}$ ", selon leur expression, qui offre matière à comparaison par rapport à leur propre mode de vie au douar. Les enfants d'Agouraï disent clairement la différence de socialisation de deux groupes, dont ils relèvent les contrastes en matière d'habillement, de comportement et de mentalité. Dans le tableau 1 nous avons retranscrit les expressions les plus significatives.

13. Nous avons réalisé deux focus group avec des enfants du primaire dans deux écoles : une urbaine et l'autre rurale. Nous voulions recueillir les commentaires des enfants dont une grande majorité a un ou plusieurs parents à l'étranger.

14. Les extraits d'entretiens résument les opinions positives des élèves vis-à-vis de l'école : "L'école nous aide à être instruits et de ne pas être analphabètes comme nos parents »; "L'école nous prépare pour l'avenir »; "L'école est bonne mais difficile à concilier avec les exigences de nos parents et les besoins d'argent de la scolarisation »; "Être à l'école est une émancipation du travail au champ "; "L'école nous aide à s'ouvrir sur le monde d'aujourd'hui »; "L'école est bien pour les filles du rural pour trouver un travail dans la ville. »

15. Nous avons aussi posé la question de savoir quelle profession ils souhaitaient exercer après leur scolarité. Les professions exercées par les proches sont fréquemment nommées : l'instituteur, le gendarme, le professeur. Viennent ensuite les professions de médecin, de juge et de journaliste. Par contre les métiers de la terre, souvent ceux des parents, n'ont pas été cités : «Le métier d'agriculture est pénible».

16. Terme utilisé dans les régions d'origine des migrants pour les désigner. Il signifie les vacanciers, les touristes, les visiteurs mais parfois tend à être utilisé péjorativement comme une insulte à l'égard des migrants. 


\begin{tabular}{|l|l|}
\hline \multicolumn{1}{|c|}{ «Voilà ce qu'ils font » } & \multicolumn{1}{c|}{ "Voilà ce qu'ils sont » } \\
\hline - parlent français & - ils sont riches \\
- ne parlent pas avec nous l'arabe ou le berbère & - ils ont des coupes de cheveux spéciales \\
- se comportent comme des invités chez nous & - ils se teignent les cheveux \\
- mangent surtout avec les fourchettes & - ils partent normaux et reviennent avec des voitu- \\
- ont plus d'argent que nous & res \\
- achètent des bonbons, de la boisson plus que & - ils s'ennuient à la maison \\
nous & - ils reviennent changés même au niveau de leur \\
& visage \\
\hline
\end{tabular}

Tableau 1 : Propos d'enfants à propos des émigrés : « ce qu'ils font » et " ce qu'ils sont » Children comments about migrants: "what they do " and « what they are"

Les enfants ont des jugements positifs à l'égard des parents en migration dès qu'ils évoquent les cadeaux qui leurs sont destinés (vêtements, jouets, fournitures scolaires, confiseries) lors des retours annuels. Ils participent aussi aux événements festifs privés et publics organisés dans l'espace villageois : repas et sorties, fêtes de famille etc., occasions justement d'observer les changements d'une année sur l'autre et de prendre la mesure des «bienfaits » de la migration tout en manifestant une certaine distance à l'égard de ces immigrés « que le temps transforme ».

Les enfants à demi-mot parlent aussi des conflits dans les familles : conflits latents et explicites qui concernent les héritages, le patrimoine familial, les aides financières, etc. Et c'est presque avec soulagement qu'ils voient les émigrés repartir pour de nombreux mois. Mais ces enfants disent aussi combien la migration est liée à la séparation des familles et peut être source de danger quand des jeunes gens tentent de franchir les frontières dans l'illégalité. Ils parlent de lahrig (brûler les frontières) et des informations que donnent les médias sur ceux qui sont morts en traversant la Méditerranée dans leur tentative de rejoindre l'Europe. Ils connaissent cette face de la migration et «sont même disposés à renoncer aux cadeaux si la migration pouvait disparâ̂tre »; "La migration ce n'est pas bien car elle sépare les enfants de leur papa »; "Elle expose les gens au danger de mort dans les bateaux »; "On peut plus faire de papiers alors pourquoi risquer sa vie »; "Ils disent que ce n'est pas comme avant maintenant en France, il n'y a pas de travail »; "La migration a été responsable du vol de bijoux de notre grand-mère par son fils »; "Lahrig ce n'est pas bien, on peut mourir »; "On peut renoncer aux cadeaux s'il faut supprimer l'émigration"; "La migration m'a séparé de ma sœur qui est partie avec ma tante »; "On ne voit plus notre père qui travaille en Italie » (Extraits d'entretiens, février 2008).

Dans un autre registre, les élèves du primaire qui n’ont jamais quitté le Maroc disent pourtant que dans les pays où résident les immigrés, les conditions de vie « sont meilleures qu'à Agouraï ». Qu'ils reproduisent le discours dominant dans leur société locale ou celui véhiculé par les médias paraboliques, ils restent convaincus «qu'ailleurs c'est mieux»: «C'est plus propre que chez nous »; "Ils sont bien éduqués »; "Ils sont plus organisés par rapport à nous »; "Ils ont plus de droits pour les enfants "; "Ils travaillent mieux que nous »; "Les femmes ont plus de droits »; "Il y a une égalité entre les femmes et les hommes "; "Les écoles sont mieux que les nôtres "; "Il y a des jardins »; "L'avenir plus garanti »; "On peut réussir plus facilement » (Extraits d'entretiens, février 2008).

Mais c'est aussi en comparant leur condition de vie d'écolier avec ceux de leurs cousines et cousins que ces jugements prennent un autre sens. Ainsi les jeunes filles terminent quasiment leur cycle scolaire en $6^{\mathrm{e}}$ à l'école du douar. Et même si les garçons le prolonge parfois de 5 à 6 mois à Agouraï ou à El Hajeb, ils arrêtent rapidement leur scolarité. Subvenir aux dépenses de location, d'alimentation et surtout déléguer à un membre de la famille qui prend en charge l'élève qui veut poursuivre ses études, représentent des dépenses que les parents ne peuvent assumer. La scolarisation devient dans ces conditions un luxe, même si elle est gratuite. Ainsi une jeune 
Zoubir Chattou, Patrick Gonin, Marie-Antoinette Hily

élève âgée de 13 ans nous dira : "c'est ma dernière année, je ne pourrai pas continuer. Déjà l'hiver quand il fait froid je ne peux pas venir à l'école et j'aide aussi mes parents ». Et pourtant cette jeune fille nous avait dit vouloir devenir avocate...

\section{Conclusion}

Les rapports qu'entretiennent les habitants avec leurs immigrés laissent apparaître des réalités complexes. Ni rejetés, ni acceptés, ni adulés, ni méprisés les immigrés occupent des positions si ce n'est contradictoires du moins paradoxales. On ne recherche pas particulièrement leur aide, mais on souhaiterait qu'elle se manifeste au-delà de leur sphère domestique, comme si l'investissement dans l'espace commun du «village » ne pouvait être que la seule condition de reconnaissance d'une promotion sociale acquise à l'étranger. Comme si l'épreuve de la migration ne pouvait trouver sanction positive que dans l'engagement de ceux qui sont partis dans une entreprise d'amélioration du bien-être local.

Finalement comment interpréter cette relation paradoxale aux émigrés-immigrés, migrants-circulants, autant de termes utilisés pour nommer ceux qui sont dans la situation qu'ils le veuillent ou non, qu'ils s'en déchargent ou non, de répondre aux souhaits des différents élus et militants associatifs. Ainsi le «bon » émigré est celui qui répond à l'attente des «villageois », celui qui n’oublie pas d'où il vient. Face à cette attente-dépendance vis-à-vis de ceux qui sont partis, les engagements des immigrés sont multiples et n'appartiennent pas aux mêmes registres : les entrepreneurs individuels sont admirés voire idolâtrés; leurs interventions créent des emplois au Maroc. Mais ces entrepreneurs sont très peu nombreux et tous les migrants n'ont pas vocation à devenir des chefs d'entreprises ou des artisans au service du développement de leur pays d'origine. D'autres migrants se cantonnent à la distribution de " cadeaux », participent à des événements festifs, distribuent des fournitures scolaires, ou équipent un centre de santé par exemple. Les initiatives collectives de migrants originaires d'une même région, à l'image de ce qui se produit dans le sud marocain (Lacroix, 2005b) n'ont pas été constatées dans cette petite ville d'Agouraï. Il semblerait que les conditions nécessaires à la réalisation de projets collectifs dans l'intérêt « villageois » n'aient pas été, ou ne sont pas encore réunies à Agouraï. En effet, les recherches conduites dans d'autres pays, dont tout particulièrement celles qui connaissent des dynamiques collectives (Gonin et al., 2008) explicitent les raisons d'un engagement pour le bien commun. L'étranger s'est installé dans une migration longue, ce qui lui a permis de s'informer, voire de se former. Les migrants engagés dans des dynamiques d'intérêt collectif pratiquent le va-et-vient et maintiennent ainsi les liens et les échanges avec ceux qui sont restés. D’autres conditions encore appartiennent au registre de la circulation migratoire des hommes et des marchandises qui favorise échanges et transferts de toutes natures. Ils doivent enfin être suffisamment nombreux et résider à proximité. Ces quatre conditions ont été pour partie réunies par les Marocains qui ont migré dans les années 1960 et 1970 ; ils étaient principalement originaires du Souss et pour une moindre mesure du Rif. Les migrants internationaux d'Agouraï sont partis plus récemment et sont relativement dispersés dans les régions de destination et les établissements restent encore précaires dans les pays où ils ont migré. Cependant et sans ignorer ces processus, des élus locaux de la Province d'El Hajeb, dont particulièrement les jeunes, souhaiteraient plus d'apport de la part des migrants dans un registre où ils pourraient devenir des bailleurs de fonds au service des projets communaux et du développement local. Les jeunes engagés dans le mouvement associatif, sans écarter cette participation, en appellent d'abord à une concertation sur les priorités des interventions à conduire et une nécessaire programmation des actions de développement où les immigrés pourraient avoir un rôle à jouer si ces actions étaient définies collectivement et plus clairement.

À Agouraï, la capacité d'expression publique s'exerce par l'intermédiaire des leaders qui optent pour une certaine vision du Maroc. Si tous s'inscrivent dans un progrès économique qui permettrait à chacun de trouver un emploi, de donner une éducation aux enfants, etc., tous par contre n'ont pas la même conception des moyens pour y parvenir et c'est bien là l'objet des malentendus 
et des oppositions. L'enjeu des dissensions porte sur « le développement » comme nous l'avons souligné et concerne les jeunes adultes, classe d'âge en situation d'accéder à des responsabilités politiques. Deux tendances se font jour, ceux qui soutiennent des projets liés aux bénéfices attendus du tourisme et ceux qui s'appuient sur l'exploitation des ressources locales et la mise en valeur de la terre. Les divergences de position idéologique et politique qui s'expriment chez les jeunes militants issus du mouvement associatif et les élus locaux témoignent aussi d'un conflit sur le mode de représentativité dans l'action entre les nouvelles élites et celles issues des structures «traditionnelles » de pouvoir. Ces conflits qui se manifestent à Agouraï témoignent aussi de l'émergence d'un espace public de discussions alimentées par les différents projets mis à l'agenda tant au niveau national que régional ou local. Dans cette logique, et comme nous l'avons montré, l'apport de la masse des immigrés à la transformation de l'espace rural n'est finalement considéré par nombre de nos interlocuteurs que comme secondaire ou marginal.

\section{Bibliographie}

Arab C., 2009. Les Aït Ayad: la circulation migratoire des Marocains entre la France, l'Espagne et l'Italie, Rennes, PUR, $360 \mathrm{p}$.

Berriane M., Hopfinger H., 1992. Migration internationale de travail et croissance urbaine dans la province de Nador (Maroc), REMI, Vol. 8, n² 2.

Charef M., 2005. Les migrations marocaines et leur relations avec le Maroc, Migrance, $\mathrm{n}^{\circ}$ 24, deuxième trimestre, p. 16-23.

Charef M., Gonin P. (dir.), 2005. Émigrés-Immigrés dans le développement local, ORMES / Sud Contact, Agadir, collection « Mobilité \& Dynamique Spatiale au Maroc», 365 p. [http://www.mshs.univ-poitiers. fr/migrinter/index.php?text=documentation/2005-agadir\&lang $=$ fr].

Chatтou Z., 1995. Les migrants marocains en Europe. Construction/reconstruction identitaire et formes d'insertion (France, Espagne, Pays- Bas). Cas des Migrations au Nord-Est du Maroc, Migrations études, $\mathrm{n}^{\circ} 64,8 \mathrm{p}$.

Dumont A., 2007. La marocanité associative en France. Militantisme et territorialité d'une appartenance exprimée à distance, Université de Poitiers, Thèse de Géographie, 624 p.

Ferrié J.-N., 1999. La gifle. Sur la mise en place d'un espace public «municipal » au Maroc, Politique africaine, $\mathrm{n}^{\circ} 74$, juin 1999, dossier Espaces publics municipaux, p. 76-83.

Gonin P., Kотьок N., Lima S., 2008. Entre réseaux et territoires, des mobilisations multiscalaires pour le développement. Réseaux migratoires et communes rurales dans la région de Kayes, Mali, Communication au colloque de l'université de Rennes, CRAPE et ESO : Espace de vie, espaces-enjeux, entre investissements ordinaires et mobilisations politiques, 5-7 novembre, $13 \mathrm{p}$.

[http://eso.cnrs.fr/TELECHARGEMENTS/colloques/rennes_11_08/Gonin_Patrick_Kotlok_Nathalie_Lima_ Stephanie.pdf]

Lacroix T., 2005a. Les réseaux marocains du développement : géographie du transnational et politique du territorial, Presses de la fondation nationale des sciences politiques, Paris, $257 \mathrm{p}$.

—, 2005b. « Les organisations de solidarité internationale issues de l'immigration marocaine : les motifs transnationaux du développement local » dans Charef (M.), Gonin (P.) (Dir.), Émigrés-Immigrés dans le développement local, Agadir, ORMES / Sud Contact, p. 191-205.

Norn D., 1970. La population rurale du Maroc, Thèse d'État de géographie, Paris, 2 vol., 279 p.; 341 p.

SCHAEFFER F., 2004. Le territoire des passe-frontières: Dynamiques sociales, identitaires et spatiales de la structuration du champ migratoire marocain, Université de Poitiers, thèse de Doctorat de Géographie, 478 p.

Cet article a été reçu le 14 juin 2009 et définitivement accepté le 30 novembre 2009. 
